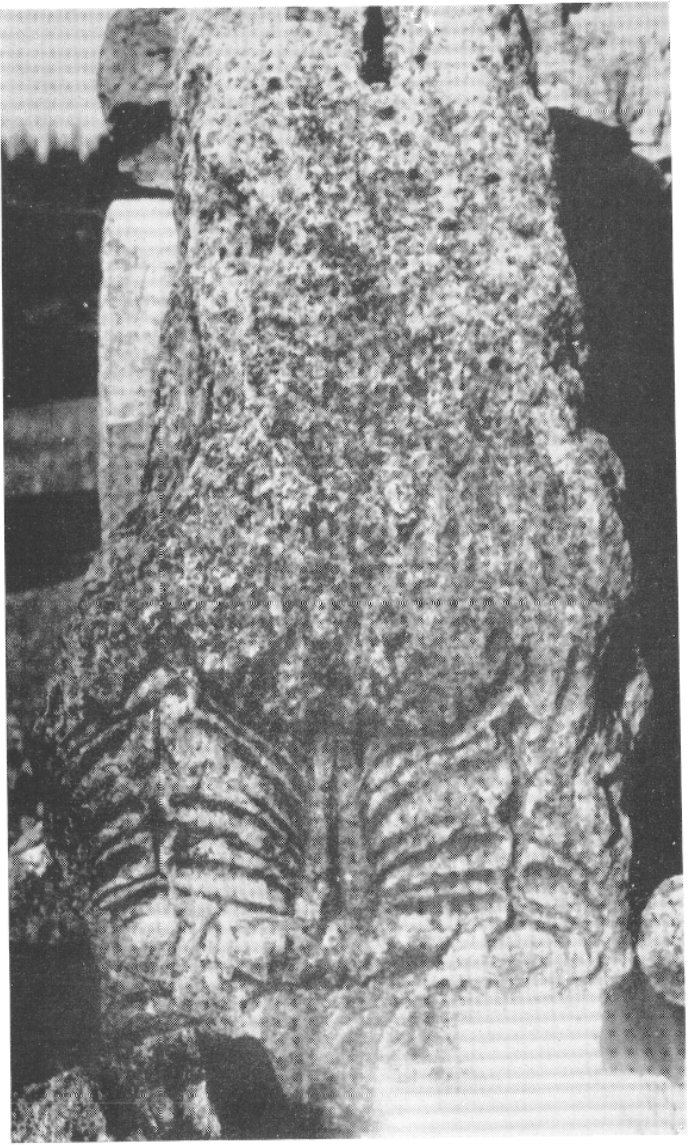

Fig. 11. - Restes d'un chapiteau de pilastre conservant les vestiges d'une décoration semblable à celle du chapiteau à figures.

architectural (fig. 11) dont on n'avail pu expliquer le rôle, et dans lequel se reconnaît aujourd'hui un chapileau de pilastre ayant offert une décoration similaire; malgré sa mutilation, on $y$ distingue trois têtes humaines se détachant entre de larges volutes, au-dessus d'une corbeille dont le même feuillage, si particulier a l'ouvre étudiée, est soutenu d'un rang de perles et pirouettes. Au même monument, èdifié dans le sanctuaire dès l'époque hellénislique, on peut vraisemblablement attribuer d'autres éléments d'archilecture rencontrés isolément au cours des fouilles, certaines bases de colonne à tores égaux et fût lisse, taillées dans une pierre de même provenance que celle employée pour le chapiteau, et peut-être aussi le couronnement en forme de linteau, décoré d'une ligne de feuillage soutenue d'un rang de perles et pirouettes, où une main inexpérimentée a plus tard creusé cinq alvéoles céphaloïdes pour la fixalion el l'exposilion d'autant de têtes coupées ${ }^{19}$.

I.e chapiteau à figures de Glanum apporte une nouvelle contribulion à l'étude de l'art. préromain dans une région que sa frontière maritime ouvrait aux influences étrangères, d'abord orientales, au siècle qui suivit la fondation de Marseille, puis italiques, quand la sicile et la Grande-Grèce tendirent à contrôler el à raréfier les échanges entre la mer Égée et Ia Méditerranće occidentale. Aux trois derniers siècles avant notre ère, les relations commerciales, concrélisées, sur le domaine massaliète, par les apports massifs de céramique campanienne, durent aussi créer avec l'Italie méridionale des contacts intellectuels el artistiques, origine de l'étroite parenté qui unit le chapiteau de Gilanum à ceux que nous venons de signaler dans la péninsule.

Henri Rolland.

(19) H. Rolnaxo, Fouilles de Glanum, II, p. 83, pl. $28, n^{0} 2$.

\title{
Le Pont romain de Ganagobie (Basses-Alpes)
}

L'étude d'un tronçon de la grande voie romaine qui, de Tarascon en Italie du nord par la vallée de la Durance et le col du MontGenèvre, prolongeait la Via Domitia, nous a donné l'occasion d'accomplir une prospection systématique de son tracé présumé entre Gavaillon et Sisteron : notre attention se portant tout particulièrement sur les nombreux cours d'eau ou torrents que la route devait traverser, nous avons eu la surprise de découvrir, caché par une luxuriante végétation mais à 300 mètres seulement d'une route nationale lrès fréquentée, un pont romain jusqu'ici inconnu, ignoré même des érudits locaux ${ }^{1}$.

(1) Cette découverte a été faite en collaboration avec M. l'Abbé P. Martel. Le monument fut alors dégagé par nos soins. Il est actuellement en instance 
Ce pont est situé sur un affluent de la Durance (rive droite), le Buès (ou Buech), petite rivière qui sert de limite aux communes de Lurs et de Ganagobie (Basses-Alpes', La roule romaine d'Alaunium (N.-D. des Anges à Lurs) à Seguslero (Sisteron) suivait à peu près, entre Giropey el Peyruis, le tracé de la route acluelle (R. N. 96-100), sur une étroite terrasse alluvionnaire entre la Durance et les collines abruptes de Lurs et de Ganagobie (fig. 1). Peu après la ferme de la (irand'Terre, pour éviter le confluent marécageux du Buès et de la Durance, la voie s'élevait et suivait une corniche taillée dans le flanc de la colline Saint-Pons, remontait sur 300 mètres environ la rive droite du Bues et, par une courbe profonde, le franchissail sur ce pont pour revenir dominer la Durance, rejoindre le chemin de Ganagobie, puis la route nationale. Ce tronçon de route en corniche fut abandonné au milieu du siècle dernier el, remplacé par un tronçon rectiligne qui traverse sur un haut remblai les abords marécageux de la Durance. Cet abandon nous a valu la conservation d'un pont romain mais aussi de la route antique sur plus d'un kilometre.

Le Buès, ruisseau de faible débit en apparence, draine en réalité un vaste bassin d'alimentation (Bois d'Aris, de Sigonce, de Lurs et de Ganagobie) susceptible de donner lieu à des crues subites; il s'est en outre creusé un lit profond, aux flancs abrupts, dont le franchissement par une route internationale nécessitait un pont.

Cet ouvrage (30 mìtres de long et 10 mètres de haut) barre la petile vallée dans toute sa largeur. Il comporle une seule arche reposant sur des culées massives qui prennent appui au nord sur le flanc de la colline de Ganagobie, au sud sur la montagne de Lurs (quartier de Saint-Pons), en un point où le rocher affleure considérablement (surtout au nord), ne laissant qu'un passage étroit au lit du Buès (fig. 2 et 4 ). Les bases de la culée nord, assises à même

de classement sur la liste des Monuments historiques. Sur la roule romaine, cf. : G. Barruol et I'. Marticl, La voie romaine de Cavaillon à Sisteron sous le HaulEmpirc. Elude lopographique, à paraître dans lu Revue d'Etudes Ligures, 1963. le roc, sont en très grand appareil pseudisodome, en calcaire de Ganagobie (mollasse marine). Épousant le pendage du rocher, cette base comporte une seule assise à l'est et trois assises à l'ouest, les parpaings n'y constituant pas des rangées régulières (fig. 3 et 5). Ces blocs font saillie sur l'édifice proprement dit ( 6 mètres de largeur hors tout) de $0 \mathrm{~m} .15$ à l'est et, par un double rejet, de $0 \mathrm{~m} .30$ à l'ouest (fig. 6). Alors que du côté nord la voûte

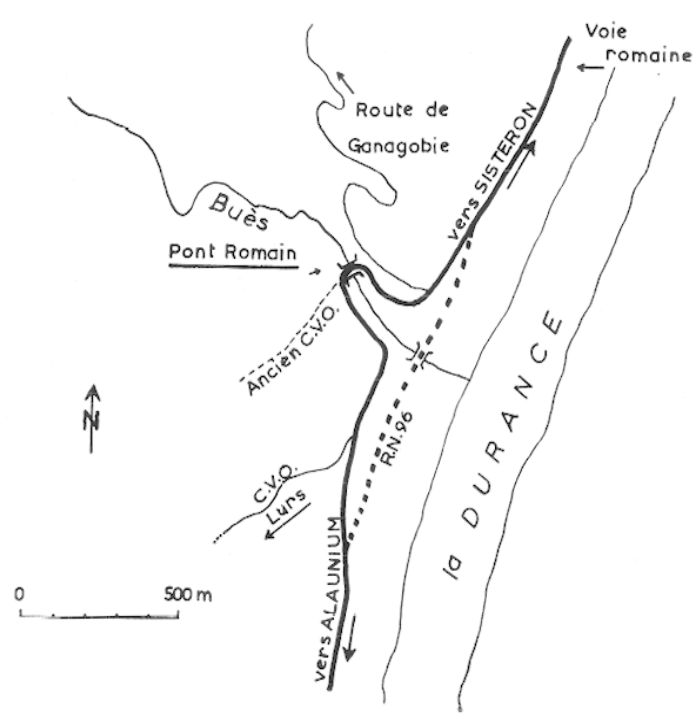

Fig. 1. - Le pont de Ganagobie : croquis de situation.

amorce directement sa courbure en plein cintre, en prenant appui sur ces fondations en grand appareil, le piédroil méridional ( 6 mètres de large et $2 \mathrm{~m} .40$ de haut) présente un parement en moyen appareil à bossages, depuis les fondations, établies sur le roc, qui affleure ici 2 mètres plus bas qu'au nord, jusqu'au départ de la voûte (fig. 2). $\Lambda$ ce piédroit, el dans son alignement, fait suite en amont un mur rectiligne de 5 mètres de long et de $0 \mathrm{~m} .80$ d'épaisseur, destiné à protéger des crues la culée sud de l'ouvrage et à canaliser le courant des eaux qui viennent battre sans cesse cette culée, car le lit sinueux de la rivière la longe en ce point bas. En aval, un mur de $3 \mathrm{~m} .20$ de long, de $0 \mathrm{~m} .80$ d'épaisseur et de $2 \mathrm{~m} .40$ de haut, joue le même rôle protecteur, mais forme un angle de $45^{n}$ par rapport à l'alignement du piédroit de la voûte (fig. 7). 


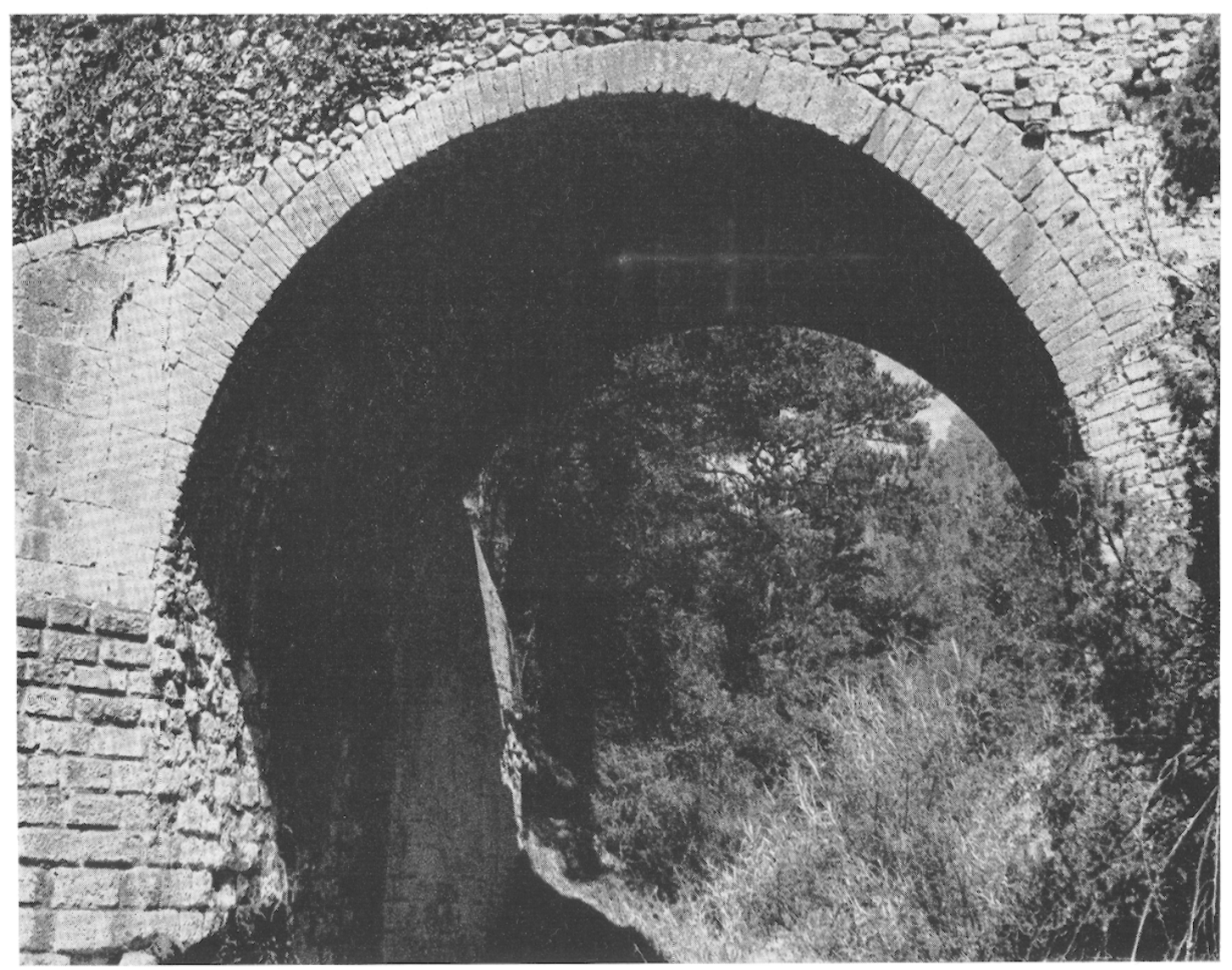

Fig. 2. - Le pont romain de Ganagobie : vue générale de la face est. Cliché J. Carré.

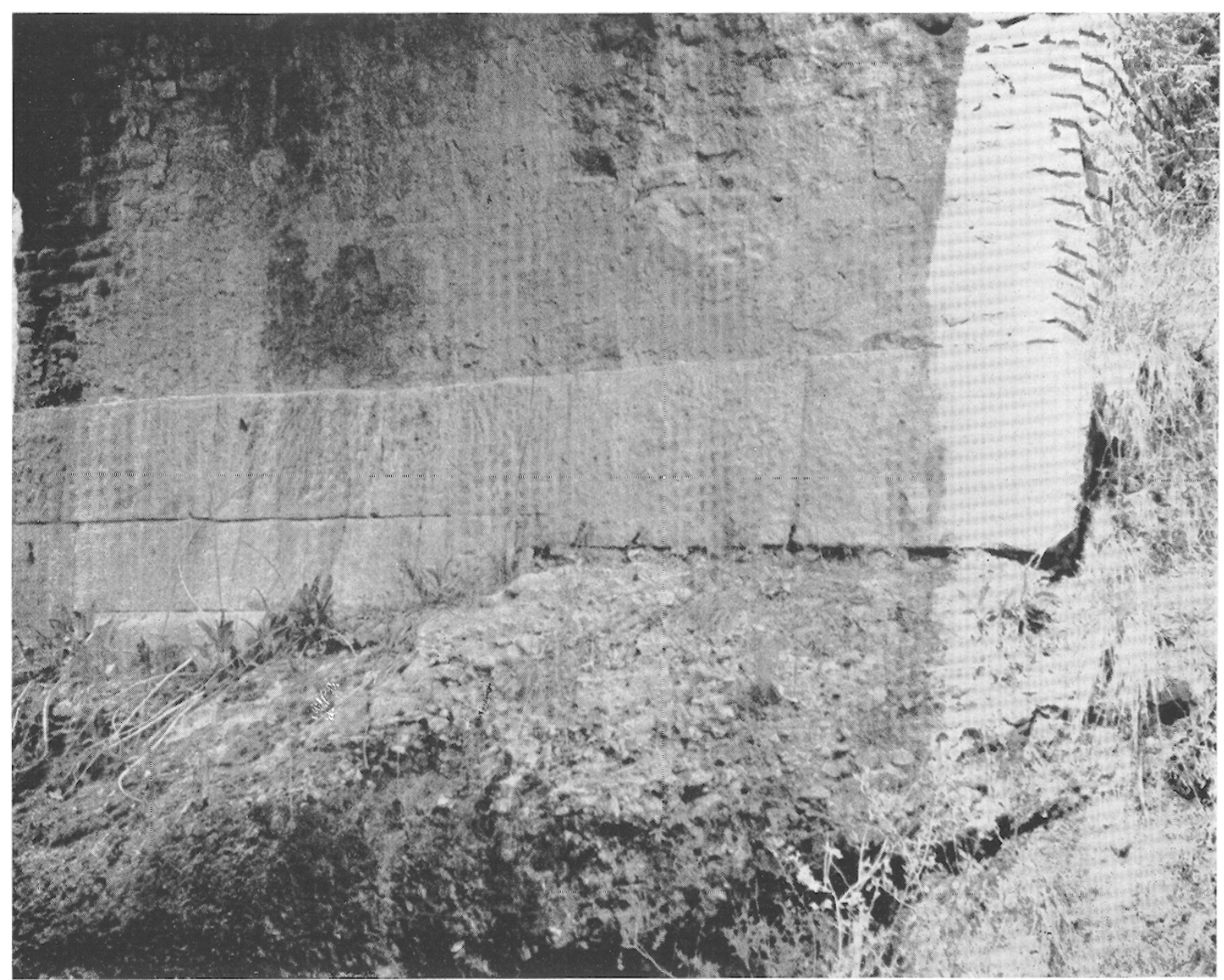

Fig. 3. - Pont de Ganagobie : base en grand appareil de la culée nord. 


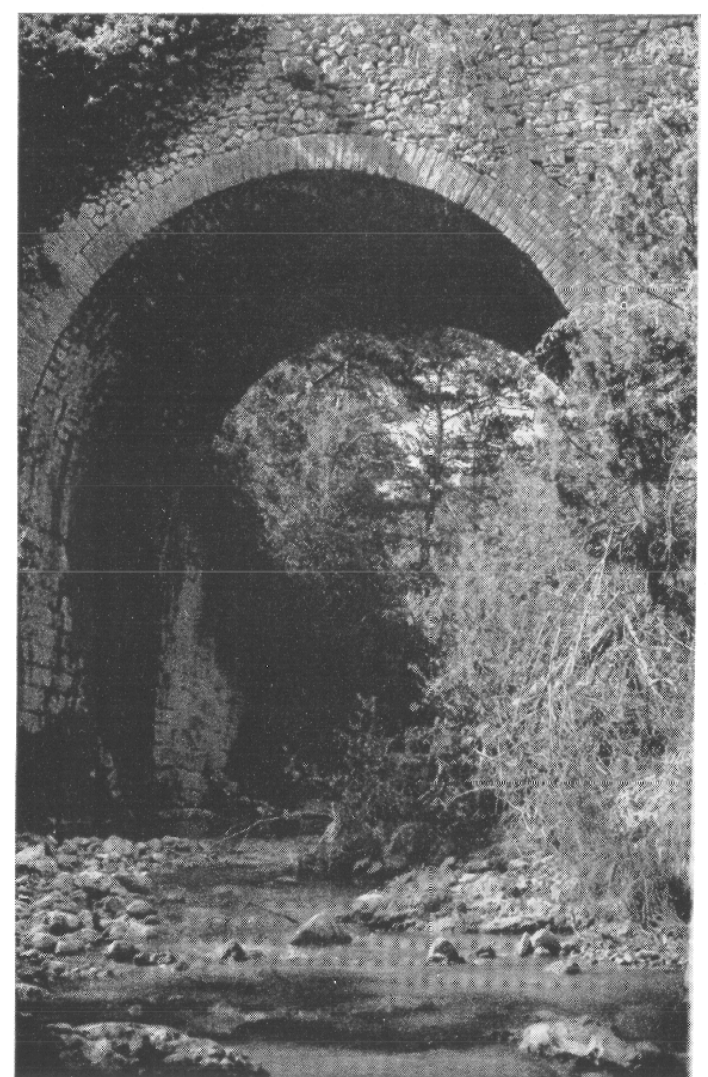

Fig. 4. - Le pont romain de Ganagobie : vue générale de la face est.

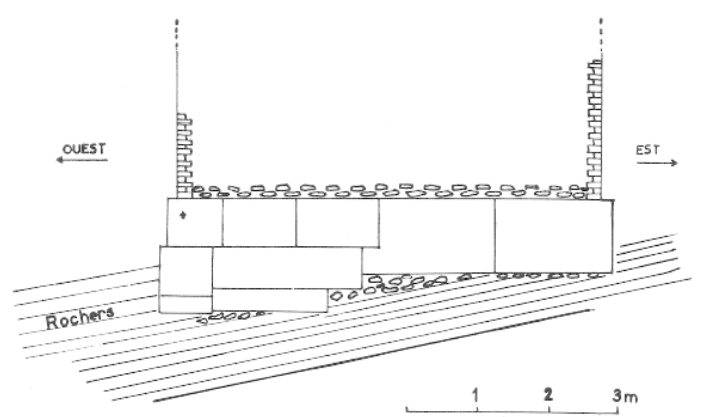

Fig. 6. - Pont de Ganagobie : élévation de la base de la culée nord.

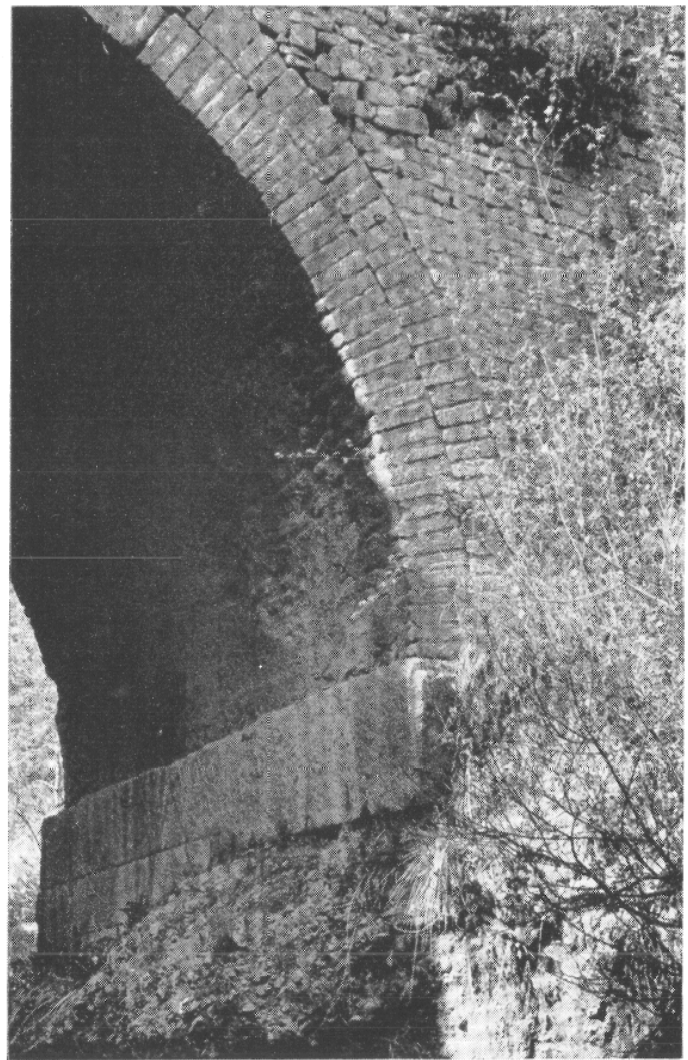

Fig. 5. - Pont de Ganagobie : culée nord.

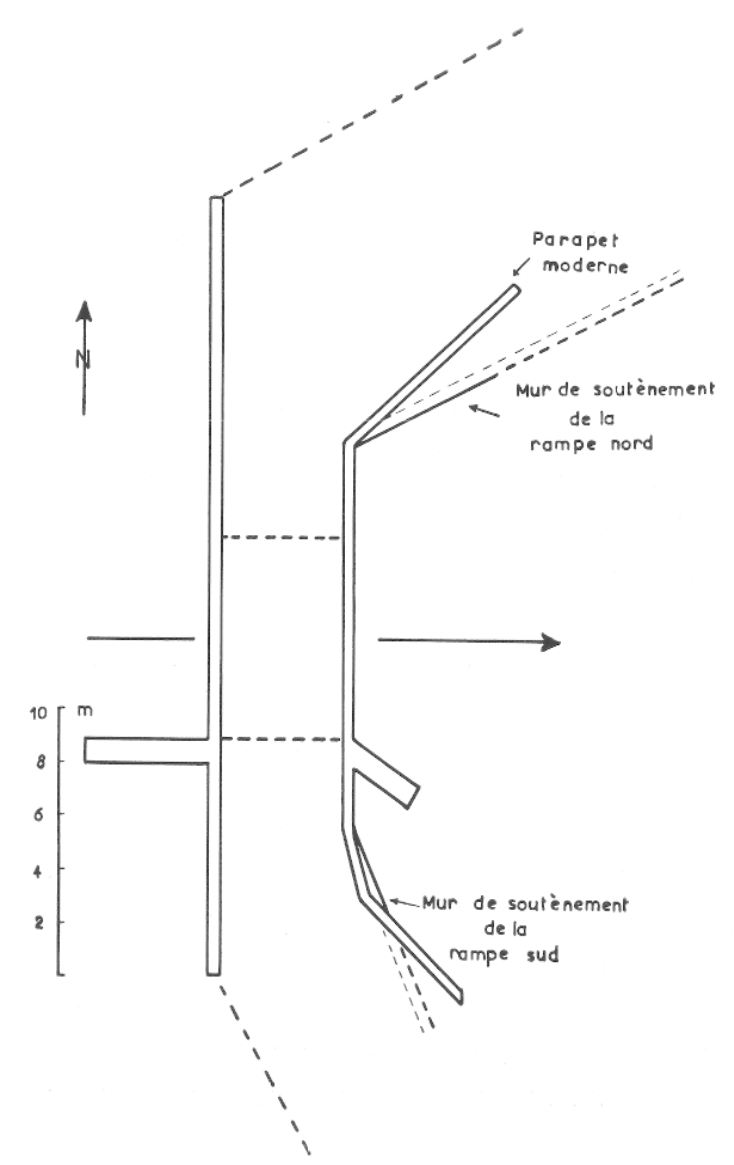

Fig. 7. - Pont de Ganagobie : $\rightarrow$ plan. Relevé de l'auteur. 


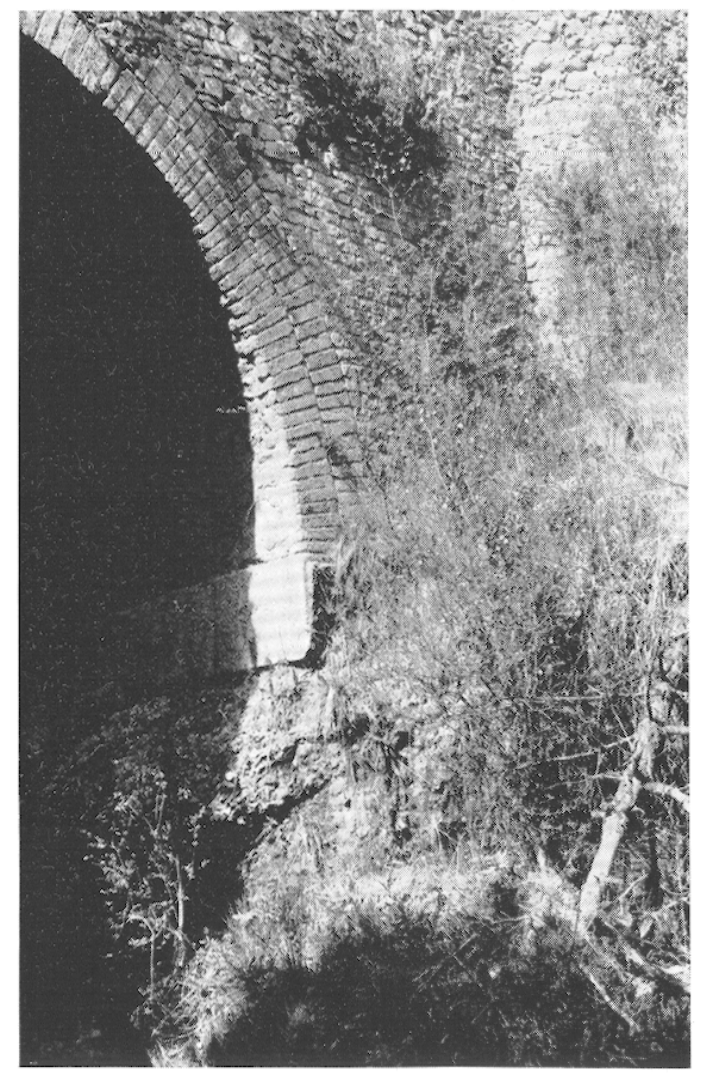

Fig. 8 : Pont de Ganagobie : archivolte de la façade orientale.

Ces deux murs protecteurs, en aval el en amont, ainsi que le piédroit de la voûte sont en moyen et grand appareil à bossages, et les assises, horizontales, sont communes à toute la longueur de ces trois éléments de murs, d'ouest en est. Mais alors que le piédroit de la voûte et. le contrefort aval présentent 13 assises, le contrefort qui les prolonge à l'ouest en comporte 16 et a de ce fail 50 centimètres de plus en hauteur. Les pierres d'appareil ont de $0 \mathrm{~m} .28$ à $0 \mathrm{~m} .30$ de hauteur el de $0 \mathrm{~m} .35$ a $0 \mathrm{~m} .65$ en longueur ; le bossage, très soigné, a un relief de 2 centimètres. Celle particularilé d'appareil contribue à donner à l'ouvrage un caractère de force et de stabilité, un caractère monumental. Les murs protecteurs, à l'est et à l'ouest, ont élé surélevés à une époque relativement récente et transformés en contreforts avec rampants pour soutenir les parlies hautes du pont (fig. 2).

L'arche a 7 mètres de hauteur sous clé et,

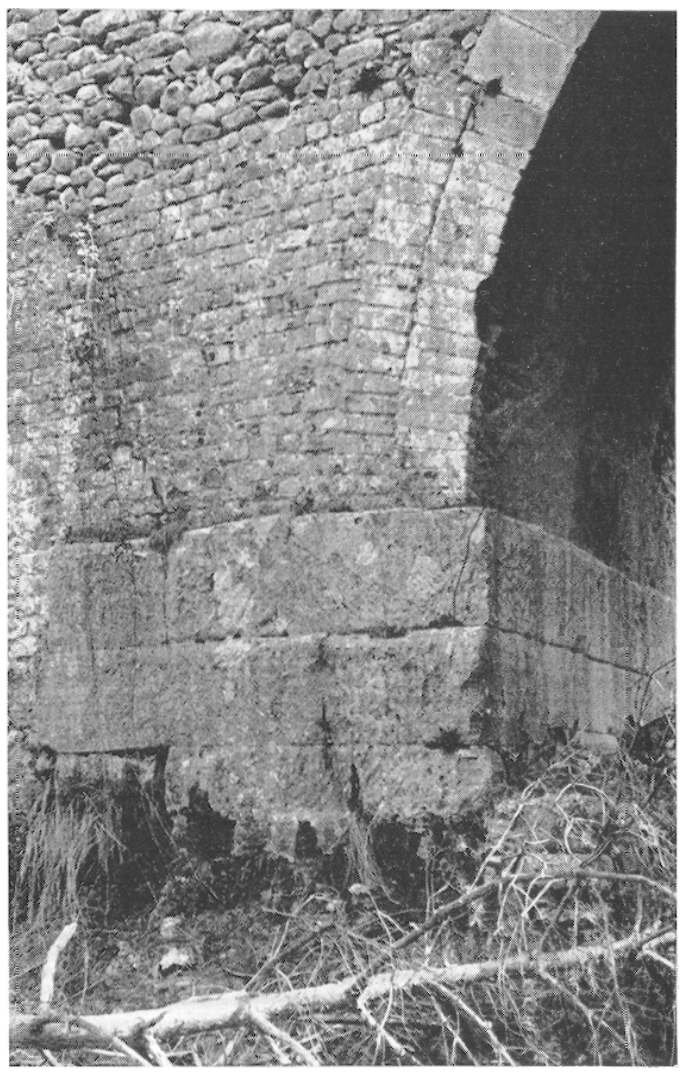

Fing. 9 ..-- Pont de Ganagobie : base de la culce nord et archivolte de la facade occidentale.

en largeur, $7 \mathrm{~m} .80$ d'ouverture au départ de la voûte. Les archivoltes des façades orientale et occidentale (malgré une importante restauration que l'on discerne aisément sur la façade ouesb) sont ornées d'un double rang de claveaux allongés en moyen appareil : au premier rang, ces claveaux ont 45) centimètres de long et, en largeur, de 11 à 14 cenlimètres à la base et de 13 à 15 centimètres à l'extrados; ils pénètrent alternalivement la voûte de 13 et de 20 centimètres (fig. 8 et 9). Les claveaux du deuxième rang n'ont que 38 centimètres de long mais sont aussi taillés de façon à converger en suivant la courbe du plein cintre. La voûte proprement dite qui supporte la chaussée (et qui est antique dans sa tolalité), est composée de claveaux convergents en moyen appareil sommairement taillés (fig. 10).

En élévation, les culćes massives, qui forment la partie la plus volumineuse du monument, sont constituées par un épais massif de 


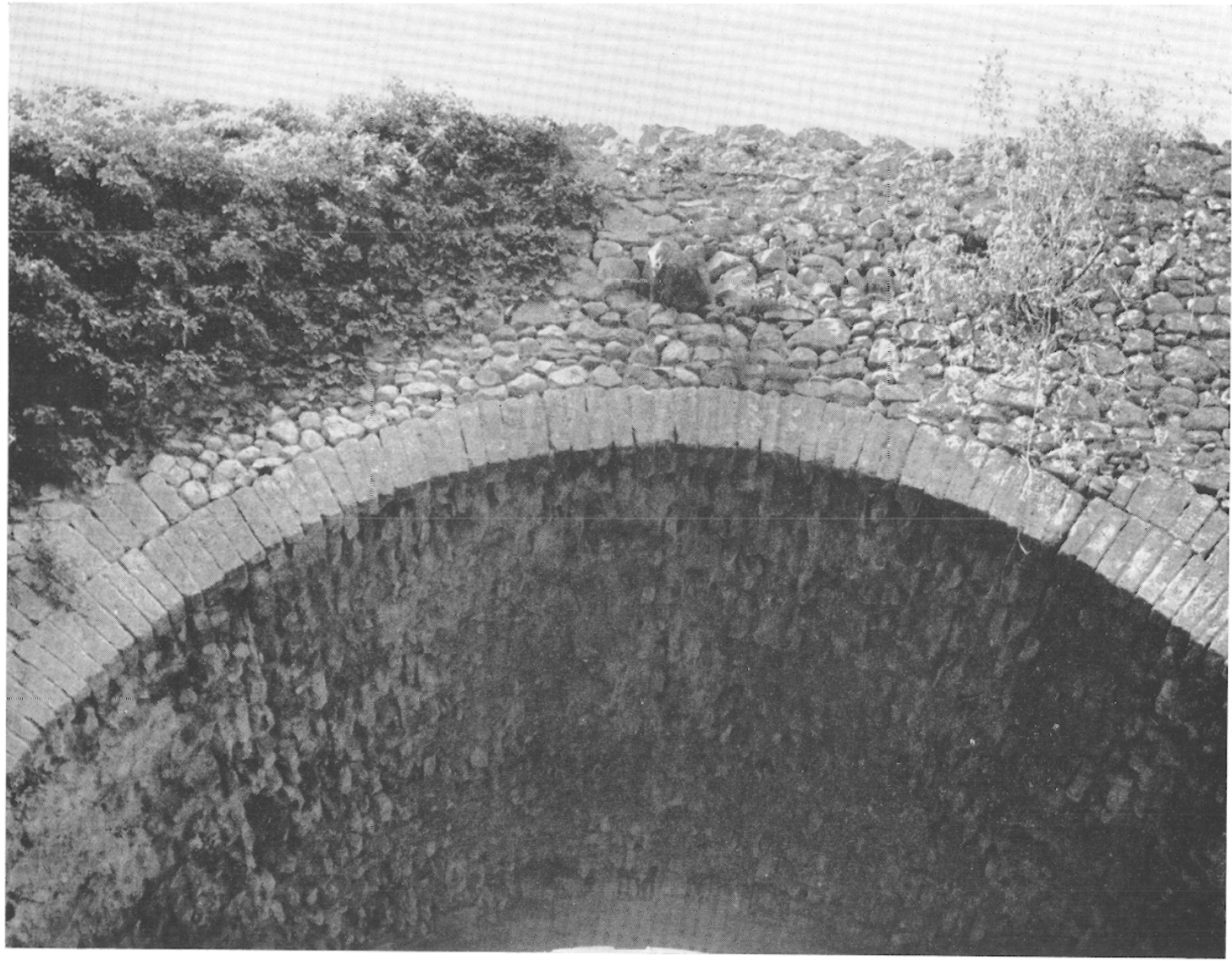

Fig. 10 - Pont de Ganagobie : voûte et archivolte orientale.

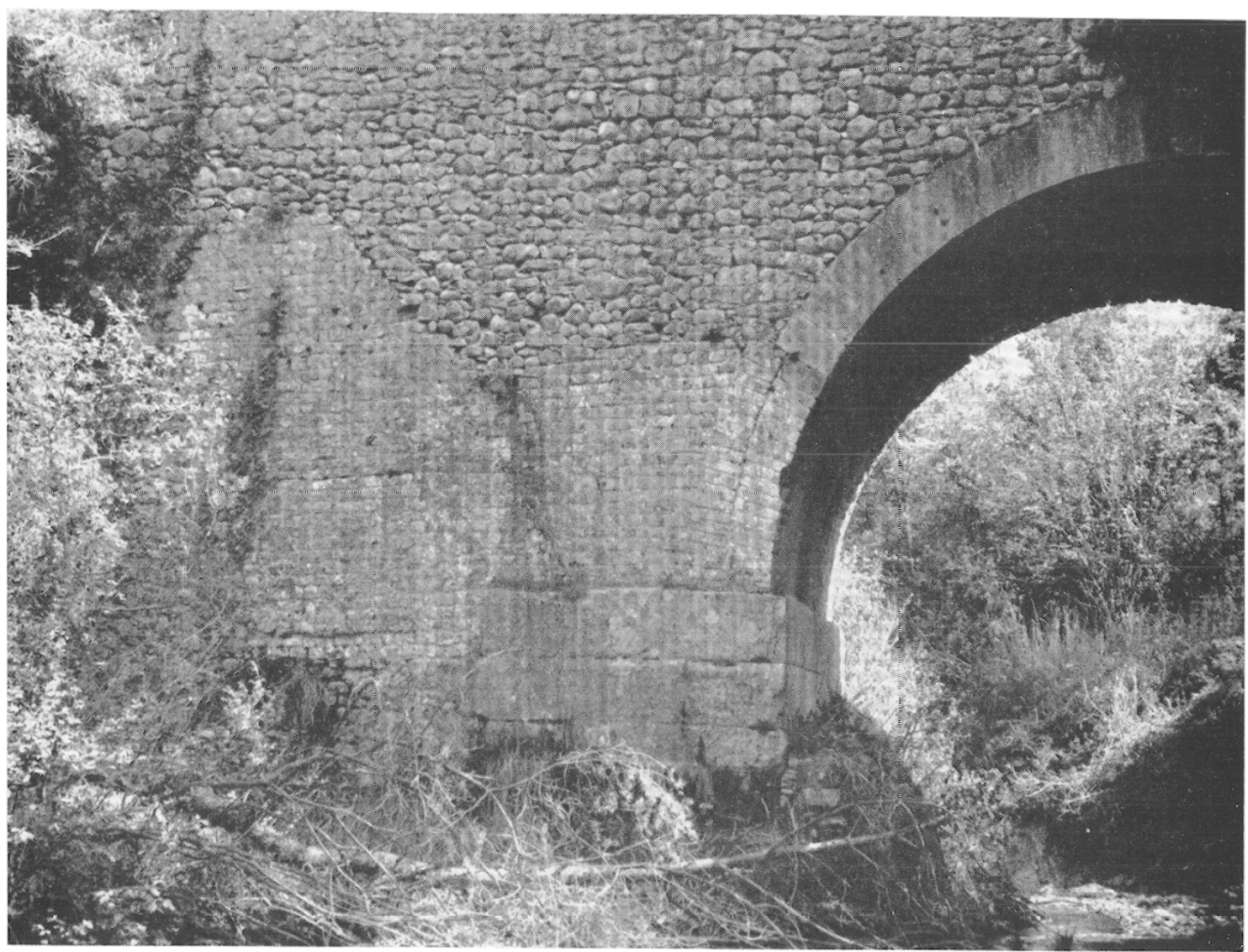

Fig. 11. - Pont de Ganagobie : parement occidental de la culée nord. 
blocage, soigneusement parementé en petit appareil sur les $2 / 3$ de sa hauteur actuelle, la partie supérieure ayant subi des restaurations au cours des siecles. On devine d'ailleurs aisément ces reprises ultérieures (fig. 10 et 11). Ce parement est constitué de petits moellons smillés très réguliers, ayant de 8 à 12 centimètres de haut et de 15 à 22 centimètres de long (calcaire barrémien des carrières locales de Saint-Donat, semble-t-il). Les lits de ce parement, disposés très régulièrement, sont horizontaux jusqu'à une certaine hauteur, mais dans les parties haules, pour épouser le léger dos d'âne que faisait la chaussée en franchissant la voûte, les assises suivent symétriquement cette déclivité : de part et d'autre du pont, un lit de moellons taillés avec une grande précision comble l'angle aigu né de cette rupture dans l'horizonlalilé des assises. Les moellons sont liés entre eux par une fine couche de ciment de 1 centimctre en moyenne. Les joints n'ont pas été repris au fer; on ne voit par ailleurs aucune trace de faux joints Lracés à la truelle, même dans les parties enterrées du monument que nous avons dégagées.

Sur la façade orientale, le parement galloromain est encore en place jusqu'au niveau de la clé de voûte ( +7 mètres); sur la face occidentale, il n'arrive qu'à mi-hauteur de l'arcade, mais descend presque jusqu'au roc (fig. 11), seules les assises inférieures, enterrées à l'origine, étant en médiocre appareil. D'une façon générale, en l'élat actuel du dégagement de l'ouvrage, c'est le parement de la culée nord, sur ses deux faces, qui est le mieux conservé. Précisons que l'emploi dans ce monument de quatre sortes d'appareils distincts n'est pas ici le fait de remaniements ou de restaurations, mais qu'il est propre à ce type de pont.

Alors que la façade occidentale de l'ouvrage est rectiligne et barre pour ainsi dire le vallon de part en part, la façade orientale présente une particularité intéressante : en effet, au nord et au sud, un mur parementé faisant un angle de $115^{\circ}$ au nord et de $155^{\circ}$ au sud avec la façade proprement dite du pont, sert de soutènement à la route sur une longueur minima de 6 mètres au nord et de 3 mètres au sud : au-delà, ces murs sont recouverts d'éboulis. Ce sont là les vestiges des longues rampes d'accès qui, faisant corps avec le pont, permettaient de maintenir la route en corniche sur les deux rives du vallon du Buès. L'ensemble du pont et de ces rampes devaient avoir un aspect monumental, qu'un dégagement complet pourrait restituer. Cet ouvrage est un exemple de la perfection des ponts et chaussées gallo-romains du réseau routier alpin.

Le parapet moderne est construit en retrait par rapport à ces murs de soutènement galloromains. La partie haute du monument, à partir de l'extrados de la voûte, a été l'objet de réparations multiples au cours des siècles : peut-être même la chaussée a-t-elle subi une surélévation par rapport au niveau antique. I'ouvrage ayant une largeur de 6 mètres hors tout, la chaussée a 5 mètres de large ; elle est bordée actuellement de chaque côté d'un parapet de $0 \mathrm{~m} .50$ d'épaisscur, mais la disposition antique devait être la même si l'on en juge d'après les ponts de ce type qui ont conservé leurs parties hautes.

Aucune décoration dans tout l'ouvrage, sinon un phallus profondément gravé sur un bloc d'angle en grand appareil de la culée nord (fig. 3) : peut-être un emblème de force, propre aux carriers, mais bien plutôt, pensonsnous, un signe apotropaïque destiné à éloigner le mal, tel qu'on le retrouve souvent dans les remparts, mais aussi sur des piles de ponts et d'aqueducs et sur d'autres grands monuments d'époque romaine ${ }^{2}$. La signification du phallus serait comparable à celle des "têtes coupées" dont la fonction apotropaïque a été démontrée

(2) G. Lugl, La lecnica edilizia romana, Rome, 1957, I, pp. 96-97, donne de nombreuses références pour l'Italie de monuments comportant ce signe. On retiendra en particulier le phallus gravé sur un ouvrage très comparable au pont de Ganagobie : l'aqueduc de Saint-Laurent d'Amasène (Marais Pontins), où il est aussi gravé sur le bloc d'angle d'un piédroit d'arcade, la partie supérieure de l'édifice étant en opus mixlum. Pour la Gaule, graflite phallique sur une pierre d'angle de grand appareil, au nord-est du monument à contrefort à Saint-Rémy (Carte archéologique de la Gaule, B.-d.-R., p. 205) ; sur l'aqueduc du Pont du Gard (ibid., Gard, 161, no 179), etc. 


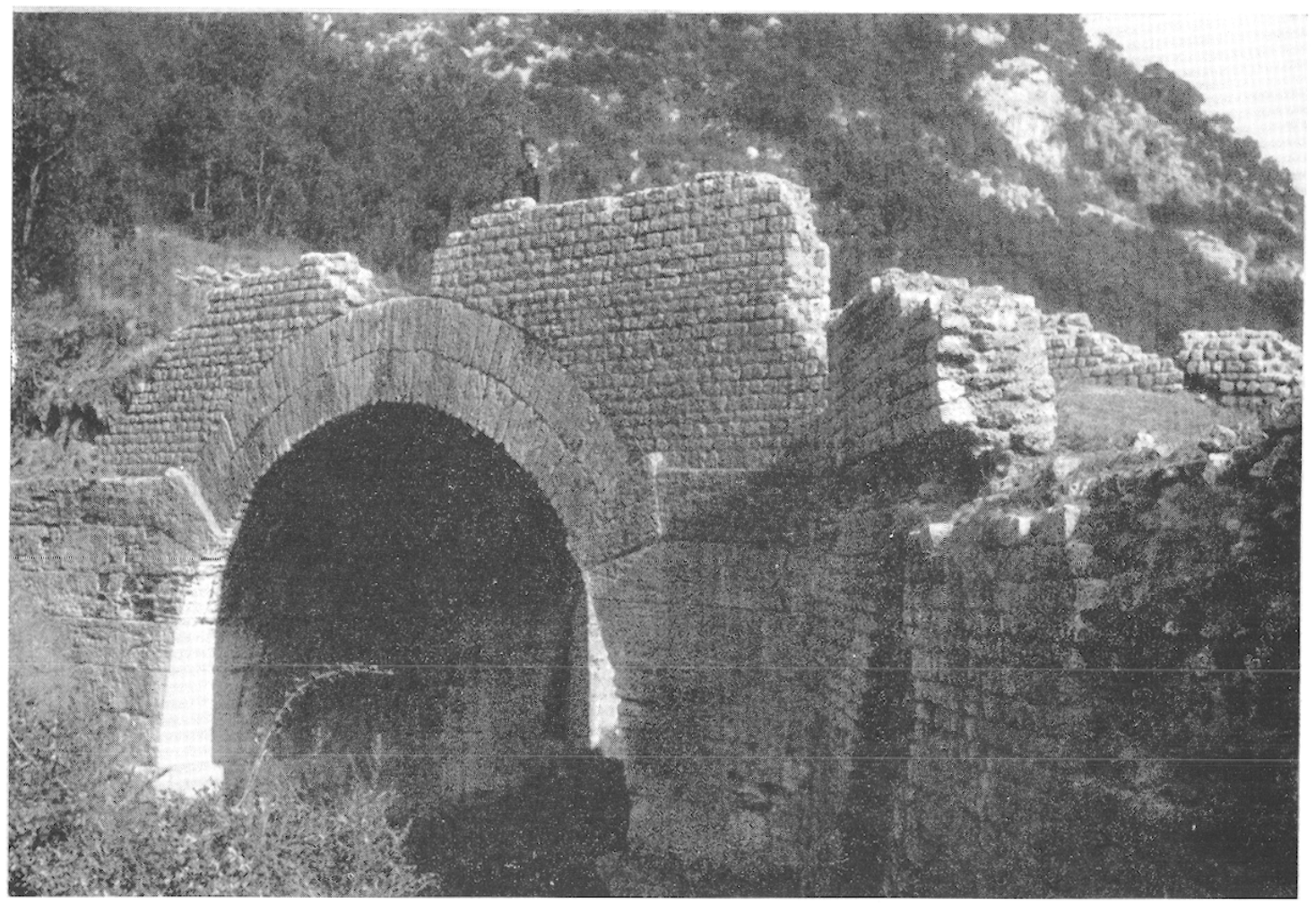

Fig. 12. - Val Ponci (Finale I.igure, I.igurie) : le pont des Fées. Cliché I.I.E.L.

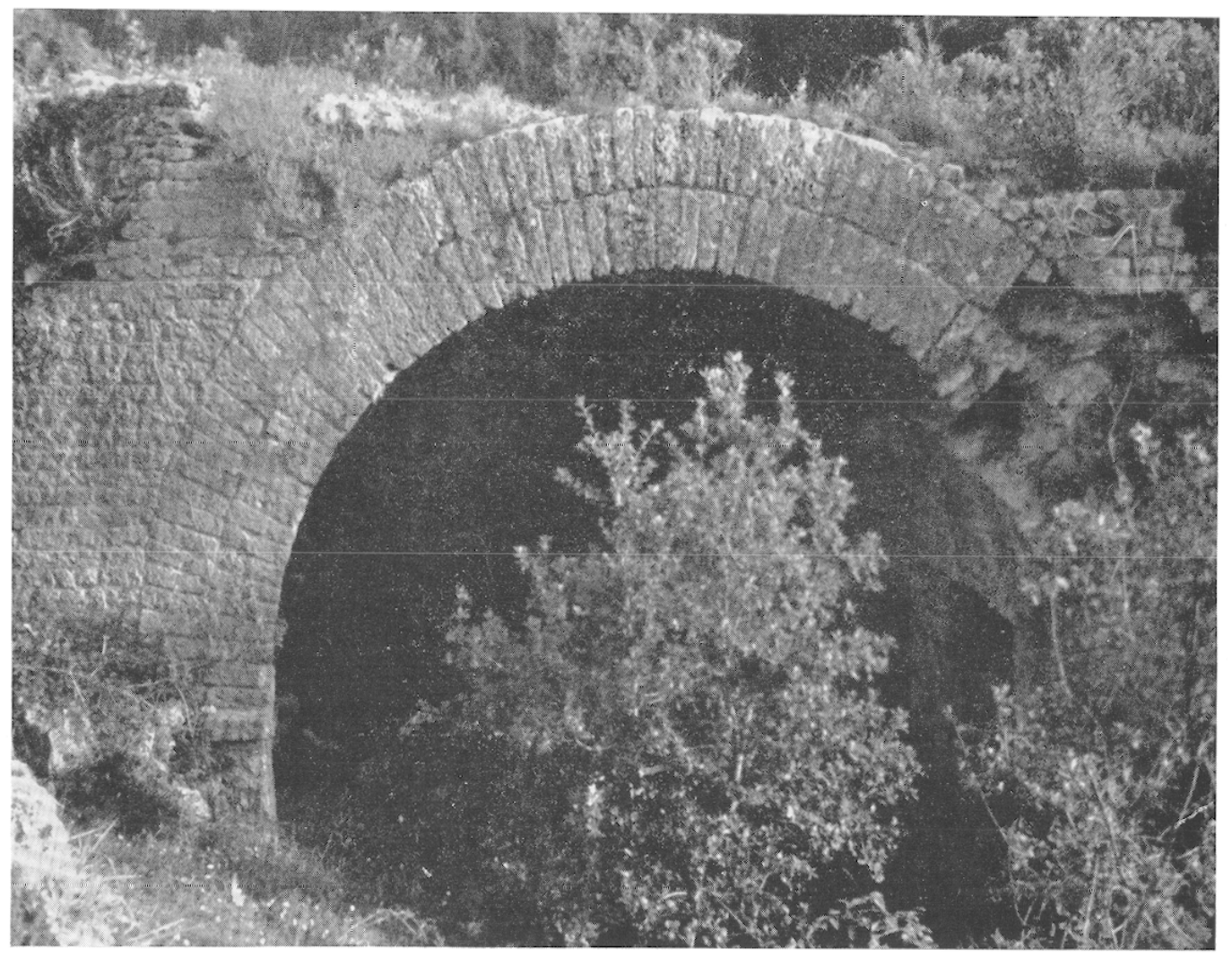

Fig. 13. - Val Ponci (Finale Ligure, Ligurie) : le pont de Voze. Cliché I.I.E.L. 
par F. Benoit ${ }^{3}$. Sur un pont, ce signe de caractère sacré symboliserait la robustesse et la stabilité d'un ouvrage prêt à faire face aux crues subites, mais aussi préserverait du mauvais œil el protégerait les voyageurs dans un passage difficile : signalons que ce pont esl aujourd'hui appelé par les habitants du voisinage "Pont de la Mort de l'Homme".

Bien que la plupart des ponts romains de Gaule Narbonnaise fussent en grand appareil, ce type de pont, parementé en petit appareil et dans lequel seuls les piédroits et les bases des culées sont en grand ou moyen appareil, n'y est pas absent. On peut rapprocher en elfel cet ouvrage du petit pont à trois arches des Esclapes, près de Fréjus, sur le canal dérivé de l'Argens ${ }^{4}$; des vestiges du pont de Villeperdrix (Drôme) récemment découvert, qui permettait à une voie secondaire de franchir un affluent de l'Eygues entre Nyons et Rémuzat ${ }^{5}$, d'un pont détruit au $\mathrm{XIX}^{\mathrm{e}}$ siècle entre Ventavon et le Monêtier-Allemont (Iautes-Alpes), sur le torrent du Bramafan, sur un autre tronçon de la grande voie de la Durance ${ }^{6}$. Il est à remarquer que tous ces ouvrages d'art sont groupés dans les Alpes, alur's qu'en Narbonnaise occidenlale on ne signale à ce jour que des ponts en grand appareil d'époque augustéenne.

Mais c'est dans les Alpes méridionales, en Ligurie, sur la via .Julia Augusla, que l'on relrouve les ouvrages les plus comparables à ceux-là : sur le torrent de Quazzola, près de Savone, sur la route romaine du Val Bormida à la mer par le col de Cadibone, où l'on voit six ponts donl, deux inlacts et encore en usage, et dans le Val Ponci près de Finale, où se trouvent cinq ponts analogues dont trois en usage : le ponl des Fées, le mieux conservé, où des fouilles récentes ont permis de dégager

(3) F. I3wort, Têles coupées de l'époque grecque all Moyen Age, dans Cahiers ligures de Préhistoire et d'Archéologie, 8, 1959, pp. 143-164.

(4) Carle arch., Var, p. 19, no 5.

(5) Gallia, XVIII, 1960, p. 372 (Informations Archeologiques).

(6) J. Roman, Répertoire archéologique des HautesAlpes, 1888, col. 115; peut-être aussi faut-il ajouter à cette liste le pont de Saint-Eusèbe, Canton de Saintl.onnet, au-delà d'Limbrun, cité par Roman, col. 140. les longues rampes d'accès (fig. 12), les ponls Sordo, delle Voze (fig. 13), dell'Acqua et di Magnone ${ }^{7}$. Ces ouvrages, auxquels il faut joindre les ponts de Loano, du Val Prino, près d'Imperia, de Porciana, près de SaintÉtienne, et de San-Remo, sur la Riviéra, ont tous les mêmes caracléristiques architecturales que le pont de Ganagobie : une seule arche en plein cintre limitée exlérieurement par un double rang de claveaux très soigneusement appareillés; un parement en petit appareil régulier enserrant un massif de blocage interne ; des bases et des murs de protection en grand appareil ; de longues rampes d'accès aménagées de façon monumentale et qui atténuent le dos d'âne, toujours marqué néanmoins; une grande largeur de voie par rapport aux ouvrages augusléens (5 à $6 \mathrm{~m}$. 50 au lieu de 3 à $3 \mathrm{~m}$. 50). Cet ensemble de ponts de Ligurie est daté du début $d u{ }_{11}{ }^{\mathrm{e}}$ siècle et serait plus précisément l'œuvre d'IIadrien qui réorganisa de façon systématique la via Julia Augusla en faisant augmenter en parliculier la largeur des ouvrages d'art.

Celte datation, confirmée en Ligurie par l'archéologie, est un point de repère qui nous permet d'assigner au pont de Ganagobie une date à peu près identique. Cet ouvrage aurait élé construit lors des restaurations de la via Domilia (tronçon alpin) entreprises sous Hadrien et continuées sous Antonin en 144145, comme en témoignent de nombreux milliaires en Languedoc et en Provence. Ces travaux des ponts et chaussées sont peut-être à mettre en rapport avec le long voyage d'Hadrien en Gaule en 121-122. On sail qu'il voyageait accompagné d'une armée d'ingénieurs et de techniciens et qu'il suscita de grands travaux publics, favorisés d'ailleurs par ses libéralités ${ }^{8}$. La réorganisation des grandes routes transalpines pouvait aussi entrer dans

(7) N. LAMboglat, Le vie romane da Vado a Loano, 1932, pp. 13-16; du même : I ponti romani di Val Quazzola, dans Rivisla Ingauna e Inlemelia, VII, 1952, p. 24; du même : Restauri e ricerche sui ponti romani di Val Ponci (Finale Ligure), dans Riv. Ing. e Inl., IX, 1954, pp. 9-14 et fig. 1-14.

(8) Dicn, LXIX, 5, 3 et 10, 1; Al:r. Victor, Ep., 14, 4-5; Hist. Aug., Jadrien, 11, 1 ; C. Jullian, IIisloire de la Gaule, IV, 1913, pp. 469-472 et notes. 
ses vues d'ordre militaire. Un document local confirme, semble-t-il, le passage de cet Empereur sur la route des Alpes par le MontGenèvre : il fit élever à Apt en effet un monument à son cheval favori Borysthène, mort sans doute au cours du voyage, et Peiresc devait retrouver au début du xvine siècle l'inscription funéraire commémorant cette anecdote de la petite histoire ${ }^{9}$.

La similitude extraordinaire qui existe entre cet ouvrage et les ponts de la via Julia Augusla dans sa traversée de la Ligurie, témoignent en outre de l'unité de vue qui devait présider au plan de réfection des grandes routes transalpines sous le règne d'Hadrien, à une époque où l'intensification du trafic, surtout sur les routes les plus fréquentées entre l'Italie et la Province Narbonnaise, nécessitait une transformation et une adaptation du réseau augustéen $^{10}$ : à savoir des chaussées plus larges, la suppression des dénivelées lrop abruptes causées par la traversée des torrents en montagnes et pour cela la construclion d'ouvrages d'art plus nombreux. Il pouvait même exister un plan-type de pont, dont les deux groupes, ligure et provençal, seraient les témoins.

(9) C.I.L., XII, 1122; inscription perdue mais considérée comme authentique. Voir aussi sur Borysthène : Hist. Aug., Iladrien, 20, 12; Dios, I.XIX, 10, 2. Peut-ĉtre le milliaire de Goult (C.I.L. XII, $5498 ;$ F.O.R. du Vaucluse, no 35 ; sur la même voie romaine du Rhône au Genèvre, est-il au nom de l'Empereur Hadrien. Une inscription trouvée à Riez (C.I.L. XII, 365) témoigne de la réfection des routes dans cette région par Hadrien, en 124 environ.

(10) Entre Cavaillon et le Mont Cenèvre, un seul pont correspond à l'aménagement de la voie des Alpes par $\Lambda$ uguste en 3 av. J.-C. : le Pont-Julien, sur le Calavon, à Bonnicux près d'Apt.
Déjà, à la fin du règne d'Auguste, Strabon ${ }^{11}$ parlant de celle grande roule qui "conduit d'Espagne en Italie ", était conscient de l'obstacle que constituait pour une route internationale la traversée des torrents alpestres : "Excellente l'été, cette route est toute fangeuse en hiver, voire au printemps. Il lui arrive même quelquefois d'être tout entière envahie et coupée par les eaux. Sans doute peut-on passer quelques-uns des fleuves que l'on rencontre à l'aide de bacs ou de ponts, bâtis soit en bois, soit en pierre, mais la grande difficulté consiste dans le passage des torrents : or il n'est pas rare de voir jusqu'à l'entrée de l'été descendre de la chaîne des Alpes des torrents d'eau que produit, la fonte des neiges. "

Pour confirmer la dalation que nous proposons, il n'est pas inutile de rappeler les similitudes architecturales qui existent entre ce type de pont et les aqueducs provençaux de Fréjus et d'Arles en particulier, dont la construction paraît remonter à la fin du I $^{\text {er }}$ et au début du II $^{\mathrm{e}}$ siècle de notre ère ${ }^{12}$ : ces ouvrages sont aussi parementés en pelits moellons réguliers et les arêtes de leurs arcs sont constituées par un rang de fins et hauts claveaux convergents.

Enfin, les différents appareils utilisés dans le pont de Ganagobie correspondent aux modes d'appareillage que l'on relrouve fréquemment a la fin $\mathrm{du} \mathrm{I}^{\mathrm{er}}$ el au second siècle en Provence intérieure.

Guy Barruol.

(11) Straeor, IV, 1, 12.

(12) Carle archéol., Var, p. 13 et B.-d.-R., no 466 (Grand Barbegal) et 478 et Plan B p. 192 ; A. Grenirer, Manuel, IV, 1, 1960, p. 81 : comme l'aqueduc d'Eygalieres, l'aqueduc de Caparon "semble appartenir aux grands travaux entrepris en Gaule après Hadrien." 